\title{
A New Fuzzy TOPSIS-TODIM Hybrid Method for Green Supplier Selection Using Fuzzy Time Function
}

\author{
Alireza Arshadi Khamseh and Mahdi Mahmoodi \\ Industrial Engineering Department, Kharazmi University, Tehran 15719-14911, Iran \\ Correspondence should be addressed to Alireza Arshadi khamseh; alireza.arshadikhamseh@gmail.com
}

Received 4 January 2014; Revised 14 February 2014; Accepted 14 February 2014; Published 17 March 2014

Academic Editor: M. Onder Efe

Copyright ( 2014 A. Arshadi khamseh and M. Mahmoodi. This is an open access article distributed under the Creative Commons Attribution License, which permits unrestricted use, distribution, and reproduction in any medium, provided the original work is properly cited.

\begin{abstract}
Today green supply chain is considered all around the world and supplier selection has been changed regarding these green and carbon emission criteria, so green supplier selection has been a major problem in this area. In this study we use fuzzy time function to assist managers in green supplier selection under uncertainty and ambiguity. This function will consider derivation from the goal during the time and by using it, and we will be able to have the best supplier in every period after having some modification in legal limitations for green supplier selection criteria. We use a fuzzy TOPSIS to have better initial weighting in TODIM, a discrete multicriteria method based on prospect theory in uncertainty (known as TODIM in Portuguese) decision making method. The results indicated that our proposed approach can easily and effectively accommodate criteria with gains and loss functions during time and also by using this method we will have a more reasonable predict of our suppliers ranking in future and that will help us in future investment in these suppliers. Finally it has been shown in car industries in Iran.
\end{abstract}

\section{Introduction}

In recent years, the European Union (EU) has established various environmental policies, including the RoHS (Restricted Use of Hazardous Substances in Electronics and Electrical Equipment) as well as WEEE (Waste Electronics and Electrical Equipment) directives. So far, environmental management has evolved to include boundary-spanning activities in the upstream and the downstream supply chains. Sirvastava defined green supply chain management (GSCM) as a combination of environmental and supply chain management activities, including product design, material selection, manufacturing process, final product delivery, and end-of-life product management. With GSCM, firms can select from a wide variety of suppliers and leverage resources throughout the firm to eliminate the environmental impact of supply chain activities, Tseng [1].

Firms typically expect their suppliers to surpass environmental compliance and to develop efficient and green product design. In addition, suppliers are expected to assess the life cycle of a product. Although the qualitative criteria are littered with subjective perception because the GSCM evolution criteria tend to be subjective, qualitative, or described with linguistic information. Thus, it is extremely difficult for the decision makers to express their preference using exact numerical values, Zhang et al. [2], so it is important to use linguistic number to distinguish between these criteria. Nevertheless, a firm's suppliers must satisfy green criteria under the constrains of subjective human preferences, which include uncertainty, in addition to uncertainties of time and planning for future; in this study we consider time in all decision making processes and this will be more effective in combination with fuzzy number for linguistic terms. Most of studies assess GSCM based on its alignment with the firm's identified objectives and fulfillment of a set of assessment criteria. In general, the evaluation of criteria is highly subjective and unstructured, as it relies significantly on manager's experience, knowledge, and intuition. However, managers cannot consider all relevant criteria due to bounded rationality and limited capacity for information processing; see Tseng [1]. The fuzzy TODIM approach can easily and effectively accommodate criteria with gains and loss functions; see Tseng et al. [3], but with all advantages of using fuzzy TODIM two problems have been remained: 


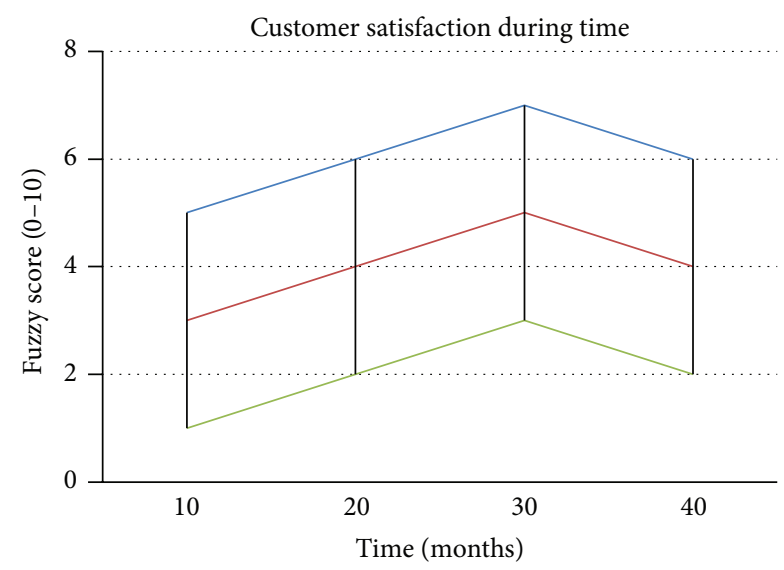

Customer satisfaction method A upper bound
Middle
Lower bound

Figure 1: Fuzzy time functions.

how can we predict our supplier behavior and how much time this supplier can be effective in our system and when we must change our supplier. Previous studies also used MADM tools for selecting suppliers according to criteria which was made it easier with using fuzzy approach. Izadikhah [4] used TOPSIS method with interval-valued fuzzy number in supplier selection and selected best supplier according to criteria; in our study, we used TODIM fuzzy because of its sensitivity to evaluate data and show how much we can improve our system with changing supplier, also we used fuzzy time function to show changes in other suppliers, and finally we can improve our supply chain with respect to this data and estimation of suppliers action. Hence, the evolution approach often implemented ineffectively because management does not effectively use appropriate knowledge and experience on GSCM. Due to the impact of this lack, management is not confident that supplier selection has been studied and applied to a set of criteria to maximize firms' green supply chain benefits. How could management apply knowledge of previously (successful and unsuccessful) supplier selections to support future decision making? The objective of this study is to create a mechanism that could assist managers in analyzing and selecting green suppliers during time horizons and this will help us to predict a picture for future of the organization activities; for these targets we use a hybrid fuzzy TOPSIS-TODIM decision making process considering fuzzy time function. We will continue this paper by literature review on fuzzy time function and fuzzy TOPSIS and also TODIM in Section 2 and also have some necessary explanations about the above techniques in Section 3; we show our proposed method in this paper by using an illustrative example for green supplier selection. Finally in Section 4 conclusions will be presented.

\section{Material and Methods}

2.1. Fuzzy Logic. A fuzzy set is a class of objects with grades of membership. A membership function is between zero and one, Zadeh [5]. Fuzzy logic is derived from fuzzy set theory to deal with reasoning that is approximate rather than precise. It allows the model to easily incorporate various subject experts' advice in developing critical parameter estimates, Zimmermann [6]. In other words, fuzzy logic enables us to handle uncertainty.

There are some kinds of fuzzy numbers. Among the various shapes of fuzzy number, triangular fuzzy number (TFN) is the most popular one. It is represented with three points as $A=\left(a_{1}, a_{2}, a_{3}\right)$. The membership function is illustrated in (1). Let $A$ and $B$ be defined as $A=\left(a_{1}, a_{2}, a_{3}\right), B=\left(b_{1}, b_{2}, b_{3}\right)$. Then $C=\left(a_{1}+b_{1}, a_{2}+b_{2}, a_{3}+b_{3}\right)$ is a sum of these two numbers. Besides, $D=\left(a_{1}-b_{3}, a_{2}-b_{2}, a_{3}-b_{1}\right)$ is the subtraction of them:

$$
\mu_{\tilde{a}}= \begin{cases}0, & x<a_{1} \\ \frac{x-a_{1}}{a_{2}-a_{1}} & a_{2} \geq x \geq a_{1} \\ \frac{a_{3}-x}{a_{3}-a_{2}} & a_{2} \geq x \geq a_{1} \\ 0, & \text { otherwise. }\end{cases}
$$

2.2. Fuzzy Time Function (FTF). Fuzzy time function is an approach to considering time as an important factor in uncertainty. In many situations we have to change our approach because of the uncertainty and changes in criteria during time. Sometimes these kinds of changes cause extra cost for organization. With fuzzy time function we could predict when some new factors like technology will be used in our system and how they could affect our decisions. de Figueiredo and Perkusich, [7] used fuzzy time function in fault and timing analysis in real time, where fuzzy time function used to verify the possibility of the input tokens in places. Yoneyama [8] used fuzzy set theory for time-delay systems that considered the uncertain Takgai-Sugeno fuzzy model with time delay. In this paper we used a new aspect of fuzzy time function which represents a combination of time and fuzzy triangular numbers.

A fuzzy time function consists of three sections, optimistic, normal, and pessimistic related to time and these lines do not cross each other's (Figure 1).

In fact, this function in each time represents a fuzzy triangular number (3), which considers all three possibilities. In many organizations some methods or some criteria will be out of date after some years or new equipment or method will be better than the older ones and these changes will effect in supplier selection methods and factors. By using FTF we could consider them in our decisions and can also upgrade our systems based on expert's viewpoints. Considering the above descriptions we will have FTF for every criterion as

$$
\operatorname{FTF}\left(c_{1}\right)= \begin{cases}F_{1} & \text { for optimistic } \\ F_{2} & \text { for normal } \\ F_{3} & \text { for pessimistic }\end{cases}
$$


According to (2) FTF represents the triangular fuzzy number during the time:

$$
\widetilde{A}=\left(a_{1}, a_{2}, a_{3}\right) \longrightarrow \widetilde{\mathrm{FTF}}=\left(F_{1}, F_{2}, F_{3}\right) .
$$

For computing the FTF in this paper we introduce two ways.

First. In this case we use average of slopes when the deviation between slopes of criteria is low and is not very important and after this we use (2) for these criteria.

To get precise decisions, it is recommended to use the separate functions for each period of time.

Second. In this method we do not use mean of the slopes because of more deviation in numbers and in these cases we use separate functions in calculations that will be more reasonable.

In some cases which fuzzy time function has more complicated function, we must use the combination of these two methods.

2.3. Fuzzy TOPSIS. TOPSIS, one of the classical multicriteria decision making methods, was developed by Hwang and Yoon [9]. It is based on the concept that the selected alternative should have the shortest distance from the positive ideal solution (PIS) and the farthest from the negative ideal solution (NIS). TOPSIS also provides an easily understandable and programmable calculation procedure. It has the ability of taking various criteria with different units into account simultaneously; see Ekmekçioĝlu et al. [10]. A number of fuzzy TOPSIS methods have been developed in recent years. Chen and Hwang [11] first applied fuzzy numbers to establish fuzzy TOPSIS method in which relative closeness for each alternative is evaluated based on fuzzy arithmetic operations. Chen [12] extends the TOPSIS method to fuzzy group decision making situations by considering triangular fuzzy numbers and defining crisp Euclidean between two fuzzy numbers. Chu [13] and Chu and Lin [14] further improved the methodology proposed by Chen [12]. Jahanshahloo et al. [15] and Chu and Lin [16] extended the fuzzy TOPSIS method based on alpha level sets with interval arithmetic. Fuzzy TOPSIS has been introduced for various multiattribute decision-making problems. Yong [17] used fuzzy TOPSIS for planet location selection, Chen et al [18] used fuzzy TOPSIS for supplier selection, and Kahraman et al. [19] used fuzzy TOPSIS to select municipal solid waste disposal method and site. Kutlu and Ekmekçioĝlu [20] used a modified fuzzy TOPSIS integrated with fuzzy AHP to propose new FMEA failure modes and effects analysis which overcomes the shortcomings of traditional FMEA. Kaya and Kahraman [21] proposed a modified fuzzy TOPSIS for the best energy technology selection.

In the following, Chen's fuzzy TOPSIS method is explained. Chen [12] extends the TOPSIS method to fuzzy group decision making situations by considering triangular fuzzy numbers and defining crisp Euclidean distance between two fuzzy numbers. In Chen's fuzzy TOPSIS, linguistic preferences can easily be converted to fuzzy numbers which
TABLE 1: Linguistic variable representing triangular fuzzy numbers.

$$
\text { Fuzzy evaluation scores for alternatives }
$$

\begin{tabular}{lc} 
Linguistic terms & Fuzzy score \\
\hline Very poor (VP) & $(0,0,1)$ \\
Poor (P) & $(0,1,3)$ \\
Medium poor (MP) & $(1,3,5)$ \\
Fair (F) & $(3,5,7)$ \\
Medium good (MG) & $(5,7,9)$ \\
Good (G) & $(7,9,10)$ \\
Very good (VG) & $(9,10,10)$ \\
\hline
\end{tabular}

are allowed to be used in calculations; see Ekmekçioĝlu et al. [10]. Kutlu and Ekmekçioĝlu, [22] introduced fuzzy FMEA using TOPSIS, and they used linguistic data for FMEA, where each of the linguistic variables represents triangular fuzzy numbers (Table 1).

In this study the linguistic variables used for each criterion and we will make FTF for each criterion during times. At the beginning weights of the criteria and fuzzy ratings of alternatives with respect to each criterion have been calculated, and the fuzzy multicriteria of decision-making problem can be expressed in matrix format as

$$
\begin{aligned}
& D=\left(\begin{array}{ccc}
x_{11} & \ldots & x_{1 n} \\
\vdots & \ddots & \vdots \\
x_{m 1} & \cdots & x_{m n}
\end{array}\right), \\
& W=\left[w_{1}, w_{2}, \ldots, w_{n}\right] \quad j=1,2, \ldots, n,
\end{aligned}
$$

where $x_{i j}$ is the rating of the alternative $A_{i}$ according to criterion $j$ (i.e., $C_{j}$ ) and $w_{j}$ denotes the importance weight of $C_{j}$. These linguistic variables can be described by triangular fuzzy numbers: $X_{i j}=\left(a_{i j}, b_{i j}, c_{i j}\right)$. To avoid the complicated normalization formula we used in classical TOPSIS, the linear scale transformation is used here to transform the various criteria scales into a comparable scale. Therefore, we can obtain the normalized fuzzy decision matrix denoted by $R$ :

$$
\widetilde{R}=\left[\widetilde{r_{i j}}\right]_{m \times n},
$$

where $B$ and $C$ are the set of benefit criteria and cost criteria, respectively, and

$$
\begin{gathered}
\tilde{r}=\left(\frac{a_{i j}}{c_{j}^{*}}, \frac{b_{i j}}{c_{j}^{*}}, \frac{c_{i j}}{c_{j}^{*}}\right), \quad j \in B ; \\
\tilde{r}=\left(\frac{a_{j}^{-}}{c_{i j}}, \frac{b_{j}^{-}}{b_{i j}}, \frac{c_{j}^{-}}{a_{i j}}\right), \quad j \in C ; \\
c_{j}^{*}=\max _{i} c_{i j} \quad \text { if } j \in B ; \\
a_{j}^{-}=\min _{i} a_{i j} \quad \text { if } i \in C .
\end{gathered}
$$

The above normalization method preserves the ranges of normalized fuzzy decision matrix as

$$
\widetilde{V}=\left[\widetilde{V_{i j}}\right]_{m \times n}, \quad i=1,2, \ldots, m ; j=1,2, \ldots, n,
$$


where

$$
\widetilde{V_{i j}}=\widetilde{r_{i j}} \cdot d\left(C_{j}\right) .
$$

According to the weighted normalized fuzzy decision matrix $\left(V_{i j}\right)$, we know that the element $\mathrm{V}_{i j}$ is positive normalized triangular fuzzy numbers and will be in $[0,1]$. Then, we can define the fuzzy positive-ideal solution (FPIS, $A^{*}$ ) and fuzzy negative-ideal solution (FPIS; $A^{-}$) as

$$
\begin{aligned}
& A^{*}=\left(v_{1}^{*}, v_{2}^{*}, v_{3}^{*}\right), \\
& A^{-}=\left(v_{1}^{-}, v_{2}^{-}, v_{3}^{-}\right),
\end{aligned}
$$

where

$$
v_{j}^{*}=(1,1,1), \quad v_{j}^{-}=(0,0,0), \quad j=1,2, \ldots, n .
$$

The distance of each alternative from $A^{*}$ and $A^{-}$can be currently calculated as

$$
\begin{aligned}
& d_{i}^{*}=\sum_{j=1}^{n} \delta\left(\widetilde{v_{i j}}, \widetilde{v_{j}^{*}}\right), \\
& d_{i}^{-}=\sum_{j=1}^{n} \delta\left(\widetilde{v_{i j}}, \widetilde{v_{j}^{-}}\right),
\end{aligned}
$$

where $d(\cdot)$ is the distance between two fuzzy numbers calculated as follows:

$$
d(\widetilde{p}, \tilde{\tau})=\sqrt{\frac{1}{3}\left[\left(p_{1}-\tau_{1}\right)^{2}+\left(p_{2}-\tau_{2}\right)^{2}+\left(p_{3}-\tau_{3}\right)^{2}\right]},
$$

where $p=\left(p_{1}, p_{2}, p_{3}\right)$ and $\tau=\left(\tau_{1}, \tau_{2}, \tau_{3}\right)$ are two triangular fuzzy numbers. The closeness coefficient of each alternative is calculated as

$$
C C_{i}=\frac{d_{j}^{-}}{d_{j}^{*}+d_{j}^{-}} .
$$

Obviously, when $C C_{i}$ is near to 1 alternative, $A_{i}$ is closer to the (FPIS, $A^{*}$ ) and farther from (FPIS, $A^{-}$). Therefore, according to the closeness coefficient, we can determine the ranking order of all alternatives and select the best one among a set of feasible alternatives.

\subsection{TODIM}

2.4.1. TODIM Method. TODIM is a discrete multicriteria method founded on prospect theory. The TODIM method has been successfully used and empirically validated in different applications. This is an experimental method based on how people make effective decisions in risky conditions. The shape of the value function of TODIM is identical to prospect theory's gain and loss function. The global multicriteria value function of TODIM aggregates all measures of gains and losses by considering all criteria. Gomes and Rangel [23] apply TODIM to investigate and recommend options for upstream projects for the natural gas reserves recently discovered in the Mexilho field in the Santos Basin, Brazil. In addition, Gomes and Rangel presented an evaluation of residential properties with real estate agents in Brazil and define a reference value for the rents of these properties' characteristics using the TODIM method for multicriteria decisions. This approach can assist professionals in the real estate market to evaluate alternatives clearly using the criteria defined by specialists. In general, TODIM can be used for qualitative and quantitative criteria. Verbal scales of qualitative criteria are converted into cardinal scales, and both types of scales are normalized. The relative measure of dominance of one alternative over another alternative is determined for each pair of alternatives. This measure is computed as the sum of all criteria of relative gain and loss values for these alternatives. This sum will be a gain, a loss, or zero, depending on the performance of each alternative with respect to each criterion. Tseng et al. [3] apply TODIM and TFNs to select green supplier chain. They used TODIM to find the best supplier, but the first weighting for TODIM with using fuzzy set theory does not consider the relationship between the criteria more obvious than fuzzy TOPSIS, with combination of these two approaches the TODIM input will be more accurate and the result will be more correct consequently. We use a new combination of TODIM and fuzzy TOPSIS and FTF in our proposed method.

In previous methods used in TODIM or other MCDM methods, data have been collected based on alternative and criteria comparisons, but we use interval-valued triangular fuzzy numbers which consider the criteria with respect to alternatives in a deterministic time and these data collection will be continued in other times which are important for decision makers, or when we have some changes in criteria or alternatives. Table 2 shows transformation of linguistic criteria to fuzzy-interval triangular numbers.

Let $A$ and $B$ be defined as TFN: $a=\left(a_{1}, a_{2}, a_{3}\right), b=$ $\left(b_{1}, b_{2}, b_{3}\right)$. The distance between $a$ and $b$ is

$$
\delta(\widetilde{a}+\widetilde{b})=\sqrt{\frac{1}{3}\left[\left(a_{1}-b_{1}\right)^{2}+\left(a_{2}-b_{2}\right)^{2}+\left(a_{3}-b_{3}\right)^{2}\right]} .
$$

The TFN is based on a three-value judgment: the minimum possible value $a_{1}$, the mean value $a_{2}$, and the maximum possible value $a_{3}$. But the interval-TFN values that have been used in this paper have 5 parameters; on the other hand these are two TFN numbers which have the same middle point (Figure 2).

$c_{i j}$ is expressed as an interval-value TFN where

$$
C=\left[\left(a_{1}, a_{1}^{\prime}\right) ; a_{2} ;\left(a_{3}^{\prime}, a_{3}\right)\right] .
$$

Given $C_{i j}=\left[\left(a_{1}, a_{1}^{\prime}\right) ; a_{2} ;\left(a_{3}^{\prime}, a_{3}\right)\right]$, the normalized performance rating is 


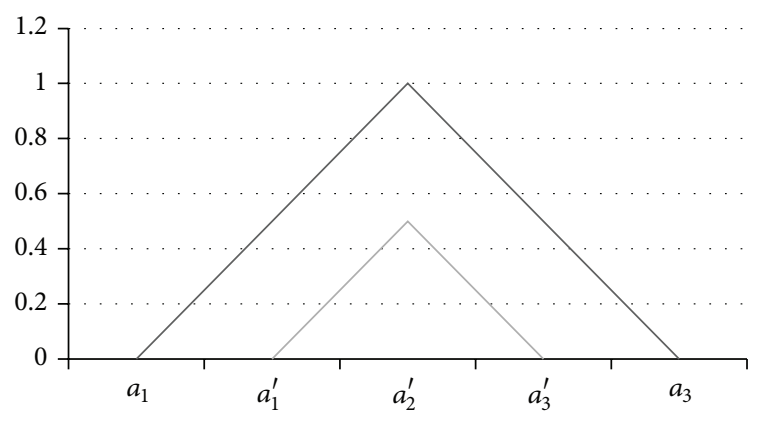

Figure 2: An interval-valued TFN.

TABLE 2: Corresponding TFNS for linguistic preferences.

\begin{tabular}{lc}
\hline Linguistic preferences & Interval-valued TFNS \\
\hline Very poor & {$[(0,0), 0,(1,1.5)]$} \\
Poor & {$[(0,0.5), 1,(2.5,3.5)]$} \\
Medium poor & {$[(0,1.5), 3,(4.5,5.5)]$} \\
Fair & {$[(2.5,3.5), 5,(6.5,7.5)]$} \\
Medium good & {$[(4.5,5.5), 7,(8,9.5)]$} \\
Good & {$[(5.5,7.5), 9,(9.5,10)]$} \\
Very good & {$[(8.5,9.5), 10,(10,10)]$} \\
\hline
\end{tabular}

$$
\begin{array}{r}
\widetilde{r_{i j}}=\left[\left(\frac{a_{i j}}{d_{j}^{+}}, \frac{a_{i j}^{\prime}}{d_{j}^{+}}\right) ; \frac{b_{i j}}{d_{j}^{+}} ;\left(\frac{a_{i j}^{\prime}}{d_{j}^{+}}, \frac{a_{i j}}{d_{j}^{+}}\right)\right], \quad i=1,2, \ldots, m ; \\
j=1,2, \ldots, n \quad \text { for } j \in I, \\
\widetilde{r_{i j}}=\left[\left(\frac{a_{j}^{-}}{d_{i j}}, \frac{a_{j}^{-}}{d_{i j}^{\prime}}\right) ; \frac{a_{j}^{-}}{b_{i j}} ;\left(\frac{a_{j}^{-}}{a_{i j}^{\prime}}, \frac{a_{j}^{-}}{a_{i j}}\right)\right], \quad i=1,2, \ldots, m ; \\
j=1,2, \ldots, n \quad \text { for } j \in J,
\end{array}
$$

where $d_{j}^{+}=\max \left\{c_{i j}, i=1, \ldots, m\right\}$ and $a_{j}^{-}=\min \left\{a_{i j}, i=\right.$ $1, \ldots, m\}, r_{i j}=\left[\left(l_{i j}, l_{i j}^{\prime}\right) ; m_{i j} ;\left(u_{i j}, u_{i j}^{\prime}\right)\right], R=\left[r_{i j}\right]_{m * n}$ and $R_{0}=\left(r_{01}, r_{02}, \ldots, r_{0 n}\right)=([(1,1) ; 1 ;(1,1)][(1,1) ; 1 ;(1,1)], \ldots$, $[(1,1) ; 1 ;(1,1)])$.

The distance between the reference value and each comparison value can be calculated by using definition (2) as follows:

$$
\begin{aligned}
& \delta_{i j}^{(1)}=\sqrt{\frac{1}{3}\left[\left(l_{i j}^{\prime}-1\right)^{2}+\left(m_{i j}-1\right)^{2}+\left(u_{i j}^{\prime}-1\right)^{2}\right]}, \\
& \delta_{i j}^{(2)}=\sqrt{\frac{1}{3}\left[\left(l_{i j}-1\right)^{2}+\left(m_{i j}-1\right)^{2}+\left(u_{i j}-1\right)^{2}\right]} .
\end{aligned}
$$

These calculations are used to determine the distance between the reference value and the comparison value in the interval; after calculation we have a new interval TFN for every TFN as $\delta_{i j}=\left[\delta_{i j}^{(1)}, \delta_{i j}^{(2)}\right]$. The weight vector of the criteria is calculated according to Zhnag et al. [2]

$$
w_{j}=\frac{\sum_{i=1}^{m}\left(\delta_{i j}^{(1)}+\delta_{i j}^{(2)}\right)}{\sum_{i=1}^{m} \sum_{i=1}^{n}\left(\delta_{i j}^{(1)}+\delta_{i j}^{(2)}\right)} .
$$

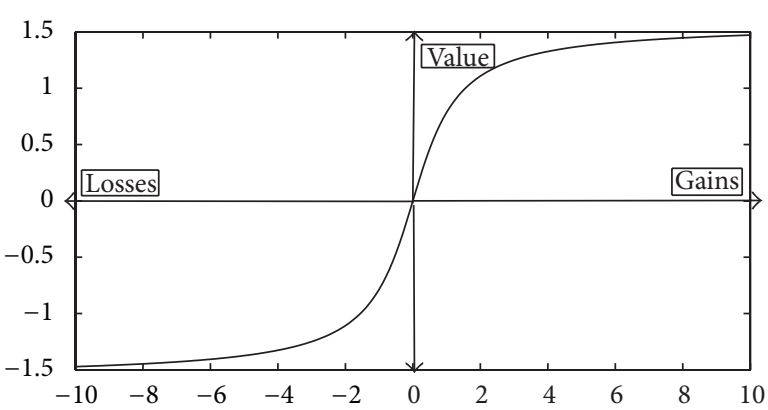

Figure 3: TODIM value function.

2.4.2. Prospect Theory. The value function used in the prospect theory is described in the form of a power law expressed as

$$
v(x)= \begin{cases}x^{\alpha}, & \text { if } x \geq 0 \\ -\theta(-x)^{\beta}, & \text { if } x<0\end{cases}
$$

where $\alpha$ and $\beta$ are parameters related to gains and losses, respectively. The parameter $\theta$ is a risk factor that is considered in model and must be greater than one. Figure 3 shows prospect value function that must be concave and has an $S$ shape form.

2.4.3. TODIM Formulation. The TODIM method uses pairwise comparisons between the criteria by using technically simple resources to eliminate occasional inconsistencies resulting from these comparisons. TODIM allows value judgments to be performed on a verbal scale using hierarchy of criteria, fuzzy value judgments, and interdependence relationships among the alternatives. The decision matrix consists of alternatives and criteria. The alternatives $A_{1}, A_{2}, \ldots, A_{m}$ are viable alternatives, $c_{1}, c_{2}, \ldots, c_{n}$ are criteria, and $x_{i j}$ indicates the rating of alternative $A_{i}$ according to the criteria $c_{j}$. The weight vector $w=\left(w_{1}, w_{2}, \ldots, w_{n}\right)$ comprises the individual weights $w_{j}(j=1, \ldots, n)$ for each criterion $c_{j}$ satisfying $\sum_{i=1}^{n} w_{j}=1$. The data of decision matrix $A$ originate from different sources. The matrix must be normalized to be dimensionless and allows various criteria to be compared with each other. This study uses the normalized decision matrix $R=\left[r_{i j}\right]_{m \times n}$ with $i=1, \ldots, m$ and $j=$ $1, \ldots, n$ :

$$
A=\left(\begin{array}{ccc}
x_{11} & \ldots & x_{1 n} \\
\vdots & \ddots & \vdots \\
x_{m 1} & \cdots & x_{m n}
\end{array}\right)
$$

TODIM then calculates the partial dominance matrices and the final dominance matrix. The first calculation that the decision makers must define is a reference criterion (typically the criterion with the greatest importance weight). Therefore, $\mathrm{w}_{r c}$ indicates the weight of the criterion $c$ by the reference criterion $r$. TODIM is expressed by the following equations. 
The dominance of an alternative over the other is as follows:

$$
\delta\left(A_{i}, A_{j}\right)=\sum_{c=1}^{m} \phi_{c}\left(A_{i}, A_{j}\right)_{\forall(i, j)}
$$

where

$$
\phi\left(A_{i}, A_{j}\right)=\left\{\begin{array}{l}
\sqrt{\frac{w_{r c}\left(x_{i c}-x_{j c}\right)}{\sum_{c=1}^{m} w_{r c}}} \\
\text { if }\left(x_{i c}-x_{j c}\right)>0, \\
0 \\
\text { if }\left(x_{i c}-x_{j c}\right)=0, \\
\frac{-1}{\theta} \sqrt{\frac{\left(\sum_{c=1}^{m} w_{r c}\right)\left(x_{i c}-x_{j c}\right)}{w_{r c}}} \\
\text { if }\left(x_{i c}-x_{j c}\right)<0 .
\end{array}\right.
$$

The term $\varphi_{c}\left(A_{i}, A_{j}\right)$ represents the contribution of criterion $c(c=1, \ldots, m)$ to the function $\delta\left(A_{i}, A_{j}\right)$ while comparing alternative $i$ with alternative $j$. The parameter $\theta$ represents the attention factor of the losses, whose mitigation depends on the specific problem. A positive $\left(x_{i c}-x_{j c}\right)$ represents a gain, whereas a nil or a negative $\left(x_{i c}-x_{j c}\right)$ represents a loss. The final matrix of dominance is obtained by summing the partial matrices of dominance for each criterion; see Tseng et al. [3]. The global value of the alternative $I$ is determined by normalizing the final matrix of dominance according to the following expression:

$$
\xi_{i}=\frac{\sum_{j=1}^{n} \delta(i, j)-\min \sum_{j=1}^{n} \delta(i, j)}{\max \sum_{j=1}^{n} \delta(i, j)-\min \sum_{j=1}^{n} \delta(i, j)} .
$$

Ordering the values $\xi_{i}$ provides the rank of each alternative, and better alternatives have higher values of $\xi_{i}$. The use of numerical values in rating alternatives may be limited in their capacity to address uncertainties. Therefore, an extension of TODIM is proposed to solve problems with uncertain data resulting in fuzzy TODIM. In practical applications, the triangular shape of the membership function is often used to represent fuzzy numbers. Fuzzy models that used TFNs proved highly effective for solving decision making problems when information is imprecise. Hence, this study provides some basic definitions of fuzzy set theory; see Tseng, [24].

In this study we first use Fuzzy TOPSIS for weighting the criteria considering time factor and then we use this data to combine with data from TODIM which calculate from comparisons between alternatives regarding to criteria, and finally we will able to determine which alternative will be more reliable and more effective in any duration of time.
TABLE 3: Criteria for green supply chain.

Annual growth in green products $\left(C_{1}\right)$

Cost of revenue: extent that it remains flat to decreases each year $\left(C_{2}\right)$

Industry leadership: green market share $\left(C_{3}\right)$

Customer retention/percentage of growth with existing customers $\left(C_{4}\right)$

Customer acquisition: the number of new green customers/total revenue to new green customers $\left(C_{5}\right)$

Life cycle assessment $\left(C_{6}\right)$

TABLE 4: Experts data collection.

\begin{tabular}{lccc}
\hline Criteria & 10 months & 20 months & 30 months \\
\hline$C_{1}$ & P, MP, VP & MP, F, MP & MG, MP, F \\
$C_{2}$ & MP, VP, F & F, MP, MP & MP, F, MG \\
$C_{3}$ & VP, F, G & MP, MP, G & F, MG, VG \\
$C_{4}$ & F, G, F & MP, VG, G & MG, G, MP \\
$C_{5}$ & G, F, P & G, VG, G & P, F, F \\
$C_{6}$ & F, MP, G & G, F, F & MP, MP, P \\
\hline
\end{tabular}

\section{Illustrative Method and Example}

3.1. Proposed Approach. We use the abovementioned method to find out the best supplier in green supply chain management considering time variations as follows (Figure 4).

Step 1. A group of decision makers identified the criteria in GSCM which are important and also will be changed during time horizons.

Step 2. Collect the opinion of decision makers with linguistic variables (Table 1) and define FTF for each important criterion.

Step 3. Use fuzzy TOPSIS to evaluate the criteria during time and initial weight of each criterion.

Step 4. Collect the opinion of decision makers on alternatives, respectively, with linguistic variables (Table 2).

Step 5. Use TODIM for evaluating the final weight of each criterion against alternatives and the relationship between them.

Step 6. Combine the results of TODIM and fuzzy TOPSIS to find out which supplier will be more effective from our company's imagination now and in the future regarding the condition changes.

\subsection{An Illustrative Example}

Step 1. In this section we study on green supplier selection problem based on time factor in a Tier company in Iran. In this company regarding the expert researches we have 6 important criteria. The data have been collected from three 


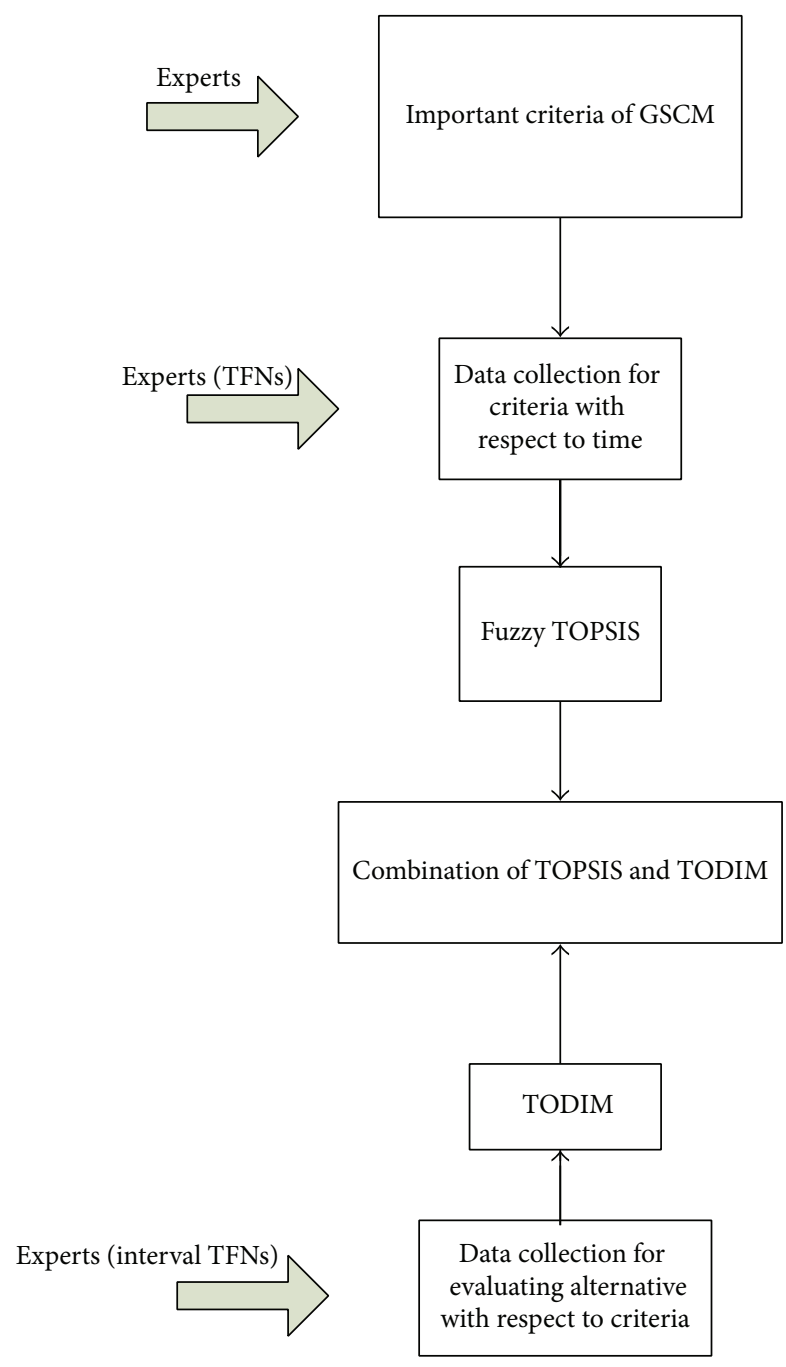

FIGURE 4: Flowchart of the method.

TABLE 5: Green supply chain criteria considering time.

\begin{tabular}{lcc}
\hline & 15 months & 25 months \\
\hline$C_{1}$ & $(3.64,5.58,7.97)$ & $(4.97,7.08,9.825)$ \\
$C_{2}$ & $(1.82,4.16,6.32)$ & $(5,8.66,8.99)$ \\
$C_{3}$ & $(2.83,5.16,6.99)$ & $(9.5,10.82,11.66)$ \\
$C_{4}$ & $(8.33,9.82,10.49)$ & $(0.33,2.84,5.51)$ \\
$C_{5}$ & $(9.82,11.4,11.6)$ & $(0,0,0)$ \\
$C_{6}$ & $(13.5,15.5,17.23)$ & $(0,0,0)$ \\
\hline
\end{tabular}

expert decision makers who have more than 10 years of experience in this area and also have the ability to predict the market and its requirements in future. Table 3 represents the criteria of GSCM which is considered in this study for our three suppliers. Table 4 represents data and then uses them to produce three functions for each criterion, but because of lack of data we consider just three points of time: 10, 20, and 30 months after data collection.
TABLE 6: Criteria weights using fuzzy TOPSIS considering time periods.

\begin{tabular}{lcc}
\hline & 15 months & 25 months \\
\hline$C_{1}$ & 0.5412 & 0.5322 \\
$C_{2}$ & 0.55 & 0.533 \\
$C_{3}$ & 0.5437 & 0.52 \\
$C_{4}$ & 0.5256 & 0.55 \\
$C_{5}$ & 0.523 & 0 \\
$C_{6}$ & 0.51 & 0 \\
\hline
\end{tabular}

TABLE 7: Criteria ranking for green supply chain considering time.

\begin{tabular}{lcc}
\hline & 15 months & 25 months \\
\hline$C_{1}$ & 3 & 3 \\
$C_{2}$ & 1 & 2 \\
$C_{3}$ & 2 & 4 \\
$C_{4}$ & 4 & 1 \\
$C_{5}$ & 5 & 5 \\
$C_{6}$ & 6 & 5 \\
\hline
\end{tabular}

Step 2. The data in Table 4 were prepared based on linguistic fuzzy variable from Table 1 , and data are collected from three experts to find out their opinion on criteria with respect to time. After collecting data with linguistic variable, we can change them to fuzzy triangular number as represented in Table 1 and then FTF must be represented. There are two methods to calculate the FTF.

First. In this case we use average of slopes, when the deviation between slopes is low and is not very important; in our example for criterion 1 we have the following.

$$
\text { Criterion } 1 \quad(0.33,1.3,3) \quad(1.6,3.6,5.6)
$$

$$
\text { pessimistic FTF }(C 1)= \begin{cases}y=0.127 t & 10<t<20 \\ y=0.14 t & 20<t<30\end{cases}
$$

then $\Longrightarrow$ pessimistic FTF $(C 1)=0.1335 t$.

The FTF from (2) for criterion 1 is

$$
\text { FTF }(C 1)= \begin{cases}y=0.185 t+5.2 & \text { optimistic } \\ y=0.15 t+3.33 & \text { normal } \\ y=0.1335 t+1.64 & \text { pessimistic. }\end{cases}
$$

To get precise decisions, it is recommended to use the separate functions for each period of time. For example for criterion 1, using the function between 10 and 20 months is more exact than considering the FTF for any time. Calculations for finding the best green supplier in our study for the 15th and 25th months are presented in Table 5; for example for Criterion 1 we have

FTF $(C 1)= \begin{cases}y=0.185 \times 15+5.2=7.975 & \text { optimistic } \\ y=0.15 \times 15+3.33=5.58 & \text { normal } \\ y=0.1335 \times 15+1.64=3.64 & \text { pessimistic. }\end{cases}$ 
TABLE 8: Interval-valued TFNs decision matrix.

\begin{tabular}{lccc}
\hline Criteria/alternative & $A_{1}$ & $A_{2}$ & $A_{3}$ \\
\hline$C_{1}$ & {$[(5.5,7.5), 9,(9.5,10)]$} & {$[(4.5,5.5), 7,(8,9.5)]$} & {$[(4.5,5.5), 7,(8,9.5)]$} \\
$C_{2}$ & {$[(5.5,7.5), 9,(9.5,10)]$} & {$[(8.5,9.5), 10,(10,10)]$} & {$[(0,1.5), 3,(4.5,5.5)]$} \\
$C_{3}$ & {$[(4.5,5.5), 7,(8,9.5)]$} & {$[(2.5,3.5), 5,(6.5,7.5)]$} & {$[(4.5,5.5), 7,(8,9.5)]$} \\
$C_{4}$ & {$[(5.5,7.5), 9,(9.5,10)]$} & {$[(0,1.5), 3,(4.5,5.5)]$} & {$[(2.5,3.5), 5,(6.5,7.5)]$} \\
$C_{5}$ & {$[(8.5,9.5), 10,(10,10)]$} & {$[(5.5,7.5), 9,(9.5,10)]$} & {$[(4.5,5.5), 7,(8,9.5)]$} \\
$C_{6}$ & {$[(4.5,5.5), 7,(8,9.5)]$} & {$[(0,0), 0,(1,1.5)]$} & {$[(8.5,9.5), 10,(10,10)]$} \\
\hline
\end{tabular}

By using FTF, we will have the variance for calculations that is useful in some other analysis which could not be achieved by ordinary triangular fuzzy numbers.

Second. In this method we did not use mean of the slopes because of more deviation in numbers and in these cases we use separate functions in calculations that will be more reasonable. For example for criterion $C 4$ we have

$$
\operatorname{FTF}(C 4)=\left\{\begin{array}{c}
\text { optimistic }\left\{\begin{array}{c}
y=0.266 t+8 \\
10<t<20 \\
y=-0.266 t+9.66 \\
20<t<30
\end{array}\right. \\
\text { normal }\left\{\begin{array}{c}
y=0.233 t+6.33 \\
10<t<20 \\
y=-0.233 t+8.66 \\
20<t<30
\end{array}\right. \\
\left\{\begin{array}{c}
y=0.166 t+4.33 \\
10<t<20 \\
y=-0.166 t+7 \\
20<t<30 .
\end{array}\right.
\end{array}\right.
$$

We use the first method for $\mathrm{C} 1$ and second method for other criteria.

Step 3. Table 5 shows that calculated numbers come from FTF for criteria; these data will be the input for fuzzy TOPSIS in (12) and (13). The results of calculations are represented in Table 6. This data will be the initial weight of TODIM approach.

By using the fuzzy TOPSIS method we have Table 6.

As shown in Table 6, we will have different ranking for criteria with respect to expert opinion changes during the time that will be measurable by FTFs. This evaluation helps us to know about the importance of each criterion in these times and gives us the perspective for more effective actions in our company in future and Table 7 presents the ranking of criteria with respect to time.

Step 4. Table 8 shows the experts' opinion on alternatives in each criteria; these data come from the linguistic variables and then change to interval valued triangular fuzzy numbers by Table 2 .
TABLE 9: Matrix of alternative scores with respect to criteria.

\begin{tabular}{lccc}
\hline & $A_{1}$ & $A_{2}$ & $A_{3}$ \\
\hline$C_{1}$ & 0.4232 & 0.6933 & 0.6933 \\
$C_{2}$ & 0.4232 & 0.1155 & 1.4598 \\
$C_{3}$ & 0.6933 & 1.0532 & 0.1155 \\
$C_{4}$ & 0.4232 & 1.4598 & 1.0532 \\
$C_{5}$ & 0.1155 & 0.4232 & 0.6933 \\
$C_{6}$ & 0.6933 & 1.9204 & 0.1155 \\
\hline
\end{tabular}

Step 5. Use TODIM methods; see Tseng et al. [3].

By applying TODIM approach, first $d^{-}, d^{*}$ have been calculated from (17) which is shown in Table 9 after that the weight of criteria has been calculated from (18) which is shown in Table 10.

After calculating weight of each criterion, the data must be normalized for calculation dominance weight of criteria. The normalized data is shown in Table 11.

Equation (23) calculates the overall value of alternative by normalizing the corresponding dominance measurements. The rank of each alternative derives from ordering the alternatives values. The global measures computed the complete rank ordering of all alternatives. In addition, a sensitivity analysis should then be applied to verify the stability of the results based on the decision makers' preferences. The results are presented in Table 12.

Considering results in Table 12, we will find out which criterion is the most important one, we can also find out ranking of alternatives regarding each criterion.

Step 6. Now the final conclusion will be conducted from Tables 12 and 6 and we can find out in next 15 months and 25 months which criteria will be more effective and finally which alternative will be the first.

By combination of Fuzzy TOPSIS which represents the weight of criteria of green supply chain with respect to time and TODIM method which shows the weight of alternatives according to criteria of supply chain we will have weights of criteria and alternatives in Tables 13 and 14.

\section{Conclusions}

This study represents a new way to select supplier during time periods by using a hybrid MCDM. Using fuzzy TOPSIS to have more accurate weighting method for TODIM and then 
TABLE 10: Criteria weights.

\begin{tabular}{ccccccc}
\hline & $C_{1}$ & $C_{2}$ & $C_{3}$ & $C_{4}$ & $C_{5}$ & $C_{6}$ \\
\hline$w_{j}$ & 0.144 & 0.159 & 0.148 & 0.233 & 0.098 & 0.217 \\
\hline
\end{tabular}

TABLE 11: Normalized scores.

\begin{tabular}{lccc}
\hline & $A_{1}$ & $A_{2}$ & $A_{3}$ \\
\hline$C_{1}$ & 0.2338 & 0.3831 & 0.3831 \\
$C_{2}$ & 0.2118 & 0.057 & 0.73 \\
$C_{3}$ & 0.3723 & 0.5657 & 0.062 \\
$C_{4}$ & 0.1441 & 0.4972 & 0.358 \\
$C_{5}$ & 0.0937 & 0.3435 & 0.562 \\
$C_{6}$ & 0.254 & 0.7037 & 0.0423 \\
\hline
\end{tabular}

TABLE 12: Final weight of criteria by using TODIM.

\begin{tabular}{lcccccc}
\hline & $C_{1}$ & $C_{2}$ & $C_{3}$ & $C_{4}$ & $C_{5}$ & $C_{6}$ \\
\hline$\xi$ & -0.59 & -0.44 & -0.99 & -0.52 & -1.083 & -0.711 \\
Normalization & 0.41 & 0.56 & 0.01 & 0.48 & 0 & 0.3 \\
\hline
\end{tabular}

Negative numbers explain losses from goal.

TABLE 13: Criteria weight's changes during time.

\begin{tabular}{ccccccc}
\hline & $C_{1}$ & $C_{2}$ & $C_{3}$ & $C_{4}$ & $C_{5}$ & $C_{6}$ \\
\hline$W, 15$ & 0.22 & 0.38 & 0.005 & 0.252 & 0 & 0.153 \\
$W, 25$ & 0.21 & 0.29 & 0.005 & 0.264 & 0 & 0 \\
\hline
\end{tabular}

TABLE 14: Alternative weights during time.

\begin{tabular}{lccc}
\hline & $A_{1}$ & $A_{2}$ & $A_{3}$ \\
\hline 15 months & 0.47 & 0.86 & 0.98 \\
25 months & 0.32 & 0.56 & 0.84 \\
\hline
\end{tabular}

combining it with fuzzy time function (FTF) helped us to rank the criteria and alternatives in several time periods. The important aim of this study was to use fuzzy time function with a new approach to consider time for each triangular fuzzy number that helped us to find out which supplier in the future according to criteria is suitable for our green supply chain and when we should change our supplier. According to the results shown in Table 14, supplier A3 has a decreasing rate from month 15 to 25 , but its decreasing rate is less than supplier 2's. Regarding these results we can assign our future improvement programs and orders to these suppliers considering criteria changes in green supplier selections and also have some repairing programs due to our vision and managerial decisions in future for these companies. Also we can assign our orders to these companies and we proposed an order plan for these suppliers regarding their portions.

\section{Conflict of Interests}

The authors declare that there is no conflict of interests regarding the publication of this paper.

\section{References}

[1] M. L. Tseng, "An assessment of cause and effect decisionmaking model for firm environmental knowledge management capacities in uncertainty," Environmental Monitoring and Assessment, vol. 161, no. 1-4, pp. 549-564, 2010.

[2] S. Zhang, S. Liu, and R. Zhai, "An extended GRA method for MCDM with interval-valued triangular fuzzy assessments and unknown weights," Computers and Industrial Engineering, vol. 61, no. 4, pp. 1336-1341, 2011.

[3] M. L. Tseng, K. H. Tan, R. J. Lina, and Y. Gengb, "Multicriteria analysis of green supply chain management using intervalvalued fuzzy TODIM," Knowledge-Based Systems, 2012.

[4] M. Izadikhah, "Group decision making process for supplier selection with TOPSIS method under interval-valued intuitionistic fuzzy numbers," Advances in Fuzzy Systems, vol. 2012, Article ID 407942, 14 pages, 2012.

[5] L. A. Zadeh, "Fuzzy sets," Information and Control, vol. 8, no. 3, pp. 338-353, 1965.

[6] H. J. Zimmermann, Fuzzy Set Theory and Its Application, International Thomson Publishing, Norwell, Mass, USA, 2001.

[7] J. C. A. de Figueiredo and A. Perkusich, "Faults and timing analysis in real-time distributed systems: a fuzzy time Petri-netbased approach," Fuzzy Sets and Systems, vol. 83, no. 2, pp. 143$168,1996$.

[8] J. Yoneyama, "Robust stability and stabilization for uncertain Takagi-Sugeno fuzzy time-delay systems," Fuzzy Sets and Systems, vol. 158, no. 2, pp. 115-134, 2007.

[9] C. L. Hwang and K. Yoon, Multiple Attributes Decision Making Methods and Applications, Springer, Berlin, 1981.

[10] M. Ekmekçioĝlu, T. Kaya, and C. Kahraman, "Fuzzy multicriteria disposal method and site selection for municipal solid waste," Waste Management, vol. 30, no. 8-9, pp. 1729-1736, 2010.

[11] S. J. Chen and C. L. Hwang, "Fuzzy multi attribute decision making," vol. 375 of lecture notes in economics and mathematical system, Springer, New York, 1992.

[12] C. Chen, "Extensions of the TOPSIS for group decision-making under fuzzy environment," Fuzzy Sets and Systems, vol. 114, no. 1, pp. 1-9, 2000.

[13] T. Chu, "Selecting plant location via a fuzzy TOPSIS approach," International Journal of Advanced Manufacturing Technology, vol. 20, no. 11, pp. 859-864, 2002.

[14] T. Chu and Y. Lin, "Improved extensions of the TOPSIS for group decision making under fuzzy environment," Journal of Information and Optimization Sciences, vol. 23273, 286 pages, 2002.

[15] G. R. Jahanshahloo, F. H. Lotfi, and M. Izadikhah, "Extension of the TOPSIS method for decision-making problems with fuzzy data," Applied Mathematics and Computation, vol. 181, no. 2, pp. 1544-1551, 2006.

[16] T. C. Chu and Y. C. Lin, "An interval arithmetic based fuzzy TOPSIS model," Expert Systems with Applications, vol. 36, no. 8, pp. 10870-10876, 2009.

[17] D. Yong, "Plant location selection based on fuzzy TOPSIS," International Journal of Advanced Manufacturing Technology, vol. 28, no. 7-8, pp. 839-844, 2006.

[18] C. T. Chen, C. T. Lin, and S. F. Huang, "A fuzzy approach for supplier evaluation and selection in supply chain management," International Journal of Production Economics, vol. 102, no. 2, pp. 289-301, 2006. 
[19] C. Kahraman, S. Çevik, N. Y. Ates, and M. Gülbay, "Fuzzy multicriteria evaluation of industrial robotic systems," Computers and Industrial Engineering, vol. 52, no. 4, pp. 414-433, 2007.

[20] A. C. Kutlu and M. Ekmekçioǧlu, "Fuzzy failure modes and effects analysis by using fuzzy TOPSIS integrated with fuzzy AHP," in Proceedings of the 1st International Symposium on Computing in Science and Engineering, pp. 706-713, 2010.

[21] T. Kaya and C. Kahraman, "Multicriteria decision making in energy planning using a modified fuzzy TOPSIS methodology," Expert Systems with Applications, vol. 38, no. 6, pp. 6577-6585, 2011.

[22] A. C. Kutlu and M. Ekmekçioğlu, "Fuzzy failure modes and effects analysis by using fuzzy TOPSIS-based fuzzy AHP," Expert Systems with Applications, vol. 39, no. 1, pp. 61-67, 2012.

[23] L. F. A. M. Gomes and L. A. D. Rangel, "An application of the TODIM method to the multicriteria rental evaluation of residential properties," European Journal of Operational Research, vol. 193, no. 1, pp. 204-211, 2009. 

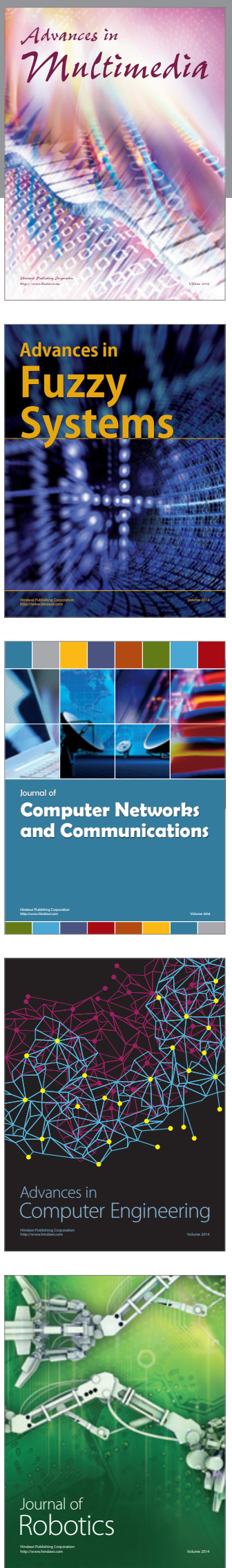

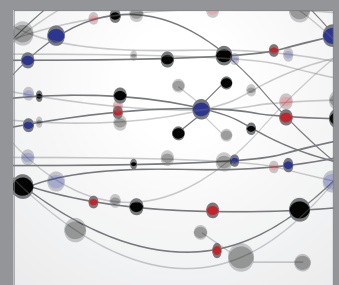

The Scientific World Journal
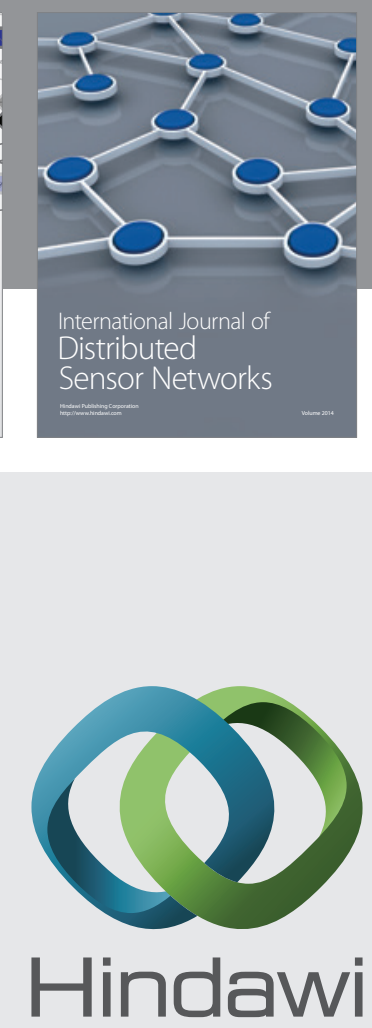

Submit your manuscripts at

http://www.hindawi.com
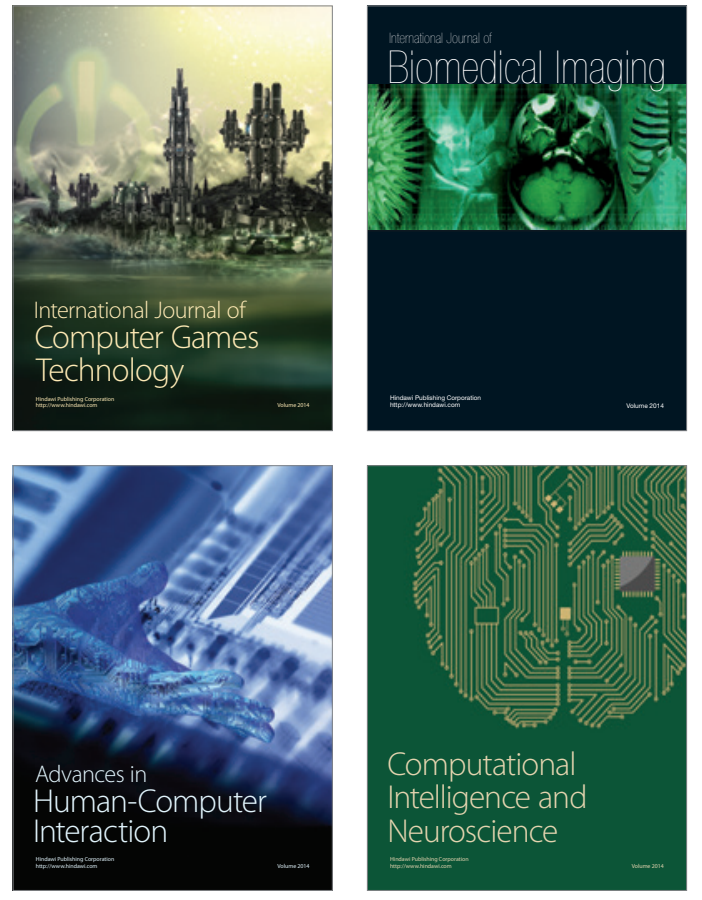
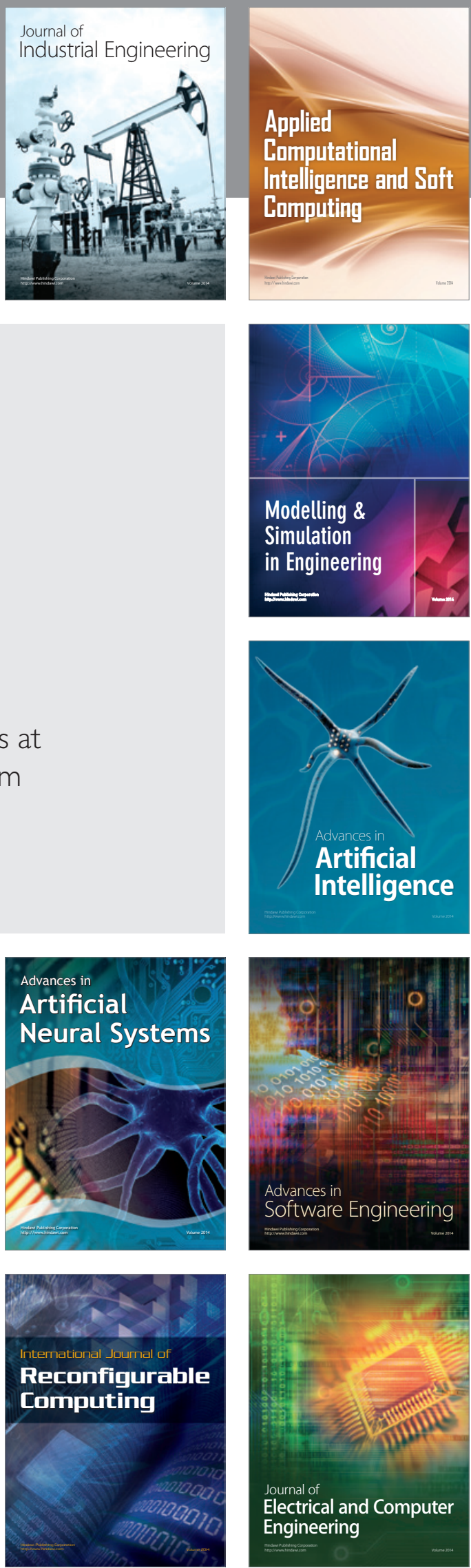